Editorial

\title{
Biomarkers and Human Biomonitoring in Occupational Medicine
}

\author{
Caterina Ledda * $\mathbb{D}$ and Venerando Rapisarda \\ Occupational Medicine, Department of Clinical and Experimental Medicine, University of Catania, \\ Via Santa Sofia 87, 95123 Catania, Italy; vrapisarda@unict.it \\ * Correspondence: cledda@unict.it; Tel.: +39-095-378-2049
}

Received: 27 August 2020; Accepted: 3 September 2020; Published: 5 September 2020

\begin{abstract}
Over the last years, biomarkers have increasingly expanded and become early and specific end points for monitoring cellular responses to various disease states and exposures to drugs and chemical agents. They have enjoyed some success as predictors of health outcomes for a number of clinical diseases, but their application for chemical exposure risk assessments has been more limited. Biomarkers may be classified into markers of exposure, effect, and susceptibility. To be useful in providing linkage to human exposure, biomarkers should be as specific as possible to the exposure agents and highly sensitive, so to detect even low levels of exposure.
\end{abstract}

Keywords: occupational medicine; biomonitoring; biomarkers; exposure; effect; susceptibility

\section{Biomarkers and Human Biomonitoring in Occupational Medicine}

Over the last years, biomarkers have increasingly expanded and become early and specific end points for monitoring cellular responses to various disease states and exposures to drugs and chemical agents [1]. They have enjoyed some success as predictors of health outcomes for a number of clinical diseases, but their application for chemical exposure risk assessments has been more limited. Biomarkers may be classified into markers of exposure, effect, and susceptibility [1].

To be useful in providing linkage to human exposure, biomarkers should be as specific as possible to the exposure agents and highly sensitive, so to detect even low levels of exposure [1-4].

Assessment methods have to be carried out in the proper biological matrix, employing analytical, reliable, and reproducible lab equipment [1-4].

Exposure biomarkers may be the exposure agent itself, chemical metabolites, products of the interaction between a chemical and some target molecules, cells, or fractions of them, detected in biological matrixes [1-5].

An aspect of the occupational medicine is evaluating the health status of workers. People who suffer a mild traumatic brain injury (mTBI) have heterogeneous symptoms and disease trajectories, which make it difficult to precisely assess long-term complications. Lee and colleagues [6], in a study, identified decreased episodic memory, anxiety, more sleep problems, and a higher global DNA methylation ratio in the blood of college students with mTBI histories. Those with multiple injuries further showed a lower processing speed than those with a single injury [6].

Shift work (SW) is associated with alterations in the human biological clock and metabolism. Serum exosomal miR-92a concentration was inversely correlated with brown adipose tissue activity playing a pivotal role in energy balance. Bracci et al. [7], in a study, focus on exosomal miR-92a as biomarker of brown adipose metabolism among shift workers. A difference in serum exosomal miR-92a levels was found in shift workers. The lower levels of miR-92a in shift workers were suggestive of a higher brown adipose tissue activity compared with daytime workers. However, the possibility that other physiological and pathological processes may influence miR-92a cannot be ruled out [7]. 
Biological risk is emerging, but the classic workplaces are often put under the "magnifying glass" and the rarer and more particular ones are left out. The pathogenicity of environmental Pseudomonas aeruginosa strains, especially isolates from museums and conservation laboratories, is not widely recognized. Rajkowska and colleagues [8] investigated on the virulence attributes of P. aeruginosa isolated from pre-Columbian textiles were compared to those of a clinical strain. Both genetically identified environmental strains (KP842564 and KP842565) exhibited a high ability to form biofilms on abiotic surfaces and high hemolytic activity. In addition, strain KP842564 was a moderate pyocyanin producer and showed proteolytic properties toward bovine serum albumin, fibrinogen, mucin, and casein. In contrast to the clinical isolate, the environmental strains were susceptible to all the tested antimicrobial agents. This study highlights the need to identify microorganisms which inhabit historic objects, in order to avoid exposure to occupational hazards [8].

Exposure limits for the general population are not always available. Salamon et al. [9] evaluate the urinary levels of free 2,5-hexanedione (2,5-HD) in Italian subjects non-occupationally exposed to n-hexane, in order to define background values in non-occupational settings. Results of this investigation highlighted that the urinary levels of free 2,5-HD were in the range of $<12.0-77.9 \mu \mathrm{g} / \mathrm{L}$ (5th-95th percentiles). The urinary excretion of the metabolite did not seem to be influenced by gender, age, smoking habit, or area of residence. Statistically significant differences $(p=0.03)$ were found between the free 2,5-HD urinary levels according to the vehicular traffic intensity within the area of residence and to body mass index of subjects. The background levels of free 2,5-HD found in this study could contribute to the definition of reference values of the general population non-occupationally exposed and could be useful to toxicologists and industrial hygienists to determine whether workers have been exposed to higher levels of n-hexane than the general population.

The influence of genetic polymorphisms of the enzymes for DNA repair and detoxification of reactive intermediates on spontaneous and bleomycin-induced (BLM) genotoxic damage in workers exposed to very low doses of ionizing radiation (IR) (mean cumulative dose $5.31 \mathrm{mSv}$ ) was studied by Stufano et al. [10]. In all the subjects examined, the frequency of chromosome aberrations (CAs) and micronuclei (MN), both spontaneous and BLM-induced, the Comet assay parameters (tail intensity), the genotypic variants of the DNA repair enzymes XRCC1 (Arg194Trp, Arg280His, Arg399Gln), XRCC3 (Thr241Met), XPD (Lys751Gln), and of the detoxification enzymes GSTM1 and GSTT1 (null genotype) and BLMH (A1450G) were determined. Among the biomarkers considered, only the frequency of total CAs $(p<0.05)$, and in particular of chromosome breaks $(p<0.01)$, was found to be significantly higher in the exposed workers than the controls. The frequency of spontaneous $\mathrm{MN}$ was higher in subjects with at least one allelic variant in XRCC1 than in carriers of the wild type, but again only in exposed workers $(p=0.046)$. Linear regression analysis showed a positive dependency of the frequency of spontaneous chromosome breaks on occupational exposure, and a dependency of the frequency of BLM-induced MN negative on occupational exposure and positive on alcohol consumption and the null GSTM1 genotype. This study points out that the frequency of chromosome breaks seems to be a useful cytogenetic biomarker for exposure to very low doses of IR, while only the combined effect of different gene variants or genetic, occupational, and lifestyle habits factors seems to be able to modulate the genotoxic effect of very low doses of IR [10].

Endocrine disruptors are an emerging chemical risk in the workplace. Kolena et al. [11] examined the occupational exposure to phthalates of hairdressing apprentices from Slovakia and the outcomes related to body composition and pulmonary functions. They observed a decrease of $\%$ of predicted values of forced vital capacity ( $\mathrm{FVC} \%$ of $\mathrm{PV}$ ) related exposure to mono(2-ethyl-5-oxohexyl) phthalate (MEOHP; $p=0.054$ ) and sum of bis(2-ethylhexyl) phthalate metabolites ( $\sum \mathrm{DEHP} ; p=0.037$ ), and a decrease of $\%$ of predicted values of vital capacity (VC\% of PV) related to exposure to MEOHP, $\sum$ DEHP ( $p=0.008$ ), and mono(2-ethyl-5-hydroxyhexyl) phthalate (MEHHP; $p=0.014$ ) in females. They detected associations between forced vital capacity (FVC) with weight $(p=0.002)$ and fat-free mass index (FFMI, $p=0.010$ ). Vital capacity (VC) and VC $\%$ of PV increased with weight, body mass index (BMI), waist circumference (WC), hip circumference (HC), waist-hip ratio (WHR), the waist-height 
ratio (WHtR), fat mass index (FMI), and FFMI in females $(p \leq 0.014)$. Results of multivariate regression between PFT and anthropometric parameters adjusted to phthalates indicated exposure to MnBP and $\mathrm{MEHP}$, changing body structure (BMI and FMI), subsequently affecting values of $\mathrm{FEV}_{1} / \mathrm{FVC}$. These data indicate that hairdressing-related phthalate exposure is significant. Moreover, biomonitoring of phthalates in this type of occupational environment is needed to regulate the harmful effect on health. Adequate attention should be given to education about potential hazards and preventive strategies in this occupational environment. An appropriate preventive strategy by pulmonary function test in periodical monitoring can detect early signs of pulmonary dysfunctions [11].

SW comprises a work schedule that involves recurring times of nonstandard work hours balanced to a fixed daily work plan with regular day work times and has been evaluated as "probably carcinogenic to humans" (Group 2A) by International Agency Research on Cancer (IARC). SW may result in increased age acceleration. Ledda and colleagues [11] in a systematic review elucidate the usefulness of telomere length as a biomarker of biological aging in shift workers. All studies analyzed underline a shortening of telomere length in SW, and aging in shift workers and duration of work. Methodologies to measure biological aging are possible to advance efforts to clarify the basic biology of aging and provide clinicians an instrument to communicate complex health advice to workers. Telomere length measures can also give an instrument for precision medicine, useful for occupational physicians in age-related screening conditions [11].

Formaldehyde (FA) is a general living and occupational pollutant, classified as carcinogenic for humans. Although genotoxicity is recognized as an FA mechanism of action, a potential contribution of epigenetic effects cannot be excluded. Leso and colleagues [12] aimed to investigate the possible epigenetic alterations induced by FA exposure in humans, animals, and cellular models with a systematic review. DNA global methylation changes were demonstrated in workers exposed to FA and also in human bronchial cells. Histone alterations, i.e., the reduction in acetylation of histone lysine residues in human lung cells were induced by FA. Moreover, a dysregulation of microRNA expression in human lung adenocarcinoma cells as well as in the nose, olfactory bulb, and white blood cells of rodents and nonhuman primates was reported. Although preliminary, these findings suggest the role of epigenetic modifications as possible FA mechanisms of action that need deeper qualitative and quantitative investigation. This may allow us to define the role of such alterations as indicators of an early biological effect and the opportunity to include such information in future risk assessment and management strategies for public and occupationally FA-exposed populations [12].

Funding: This research received no external funding.

Conflicts of Interest: The authors declare no conflict of interest.

\section{References}

1. Jakubowski, M.; Trzcinka-Ochocka, M. Biological Monitoring of Exposure: Trends and Key Developments. J. Occup. Health 2005, 47, 22-48. [CrossRef] [PubMed]

2. Koh, D.S.; Koh, G.C. The use of salivary biomarkers in occupational and environmental medicine. Occup. Environ. Med. 2007, 64, 202-210. [CrossRef]

3. Hirvonen, A. Genetic Factors in Individual Responses to Environmental Exposures. J. Occup. Environ. Med. 1995, 37, 37-43. [CrossRef] [PubMed]

4. Angerer, J.; Ewers, U.; Wilhelm, M. Human biomonitoring: State of the art. Int. J. Hyg. Environ. Health 2007, 210, 201-228. [CrossRef] [PubMed]

5. Ledda, C.; Senia, P.; Rapisarda, V. Biomarkers for Early Diagnosis and Prognosis of Malignant Pleural Mesothelioma: The Quest Goes on. Cancers 2018, 10, 203. [CrossRef]

6. Lee, H.; Lee, S.; Black, I.; Salado, L.; Estrada, J.; Isla, K. Long-Term Impact of Mild Traumatic Brain Injuries on Multiple Functional Outcomes and Epigenetics: A Pilot Study with College Students. Appl. Sci. 2020, 10, 4131. [CrossRef]

7. Bracci, M.; Elexpuru-Zabaleta, M.; Tartaglione, M.F.; Ledda, C.; Rapisarda, V.; Santarelli, L. Exosomal miR-92a Concentration in the Serum of Shift Workers. Appl. Sci. 2020, 10, 430. [CrossRef] 
8. Rajkowska, K.; Otlewska, A.; Guiamet, P.S.; Wrzosek, H.; MacHnowski, W. Pre-Columbian Archeological Textiles: A Source of Pseudomonas aeruginosa with Virulence Attributes. Appl. Sci. 2019, 10, 116. [CrossRef]

9. Stufano, A.; Chiarappa, P.; Bagnulo, R.; Drago, I.; Rapisarda, V.; Ledda, C.; Vimercati, L.; De Benedictis, L.; Resta, N.; Soleo, L.; et al. Influence of Polymorphisms of DNA Repair and GST Genes on Genotoxic Damage and Mutagen Sensitivity in Workers Occupationally Exposed to Very Low Doses of Ionizing Radiation. Appl. Sci. 2019, 9, 5175. [CrossRef]

10. Kolena, B.; Petrovičová, I.; Sidlovska, M.; Hlisnikova, H.; Tomasovova, E.; Zoldakova, V.; Trajtelova, H.; Rybansky, L.; Wimmerova, S.; Trnovec, T. Phthalates Exposure and Occupational Symptoms among Slovakian Hairdressing Apprentices. Appl. Sci. 2019, 9, 3321. [CrossRef]

11. Ledda, C.; Loreto, C.; Rapisarda, V. Telomere length as a biomarker of biological aging in shiftworkers. Appl. Sci. 2020, 10, 2764. [CrossRef]

12. Leso, V.; Macrini, M.C.; Russo, F.; Iavicoli, I. Formaldehyde Exposure and Epigenetic Effects: A Systematic Review. Appl. Sci. 2020, 10, 2319. [CrossRef]

(C) 2020 by the authors. Licensee MDPI, Basel, Switzerland. This article is an open access article distributed under the terms and conditions of the Creative Commons Attribution (CC BY) license (http://creativecommons.org/licenses/by/4.0/). 